\title{
On the Compliance of Coiled Springs
}

\author{
X. Ding, \\ Robotics Research Institute, \\ Beijing University of Aero.\& Astro., \\ Haidian District Beijing 100083 \\ P.R. China \\ and \\ J.M. Selig* \\ Faculty of Business, Computing and Information Management \\ London South Bank University \\ London SE1 0AA, U.K.
}

\begin{abstract}
The $6 \times 6$ spatial compliance matrix for a helical spring is computed. The method used is to find the compliance of infinitesimal elements along the length of the spring. Then integration is used to sum the compliances and produce the total compliance of the spring. A key point in the method is that the compliance matrix for each element must be expressed in a common coordinate frame.
\end{abstract}

*e-mail: seligjm@1sbu.ac.uk 
The results produced are significantly different from the those given in elementary texts. However, it is shown how these results can be recovered by making the standard "closely coiled" approximation. Next, the principal wrenches and eigencompliances of the spring are studied, both for the closely coiled spring and for the case where these assumptions are not made. Finally some numerical examples are given.

Keywords Coiled springs, Spatial Compliance, Principal Stiffnesses.

\section{Introduction}

In robotics and mechanism theory it is common to consider the compliance or stiffness properties of the system. Although for accurate work there exists a wealth of finite element computer packages which can determine the behaviour of compliant elements, it is still important to consider the first order properties determined by the compliance matrix. For many robots and mechanisms traditionally stiff materials such as steel are used; and designs deliberately try to maximize stiffness and eliminate vibrations. In such cases an understanding of the behaviour for small deflections is normally sufficient.

Historically Ball [1] was probably the first to study the stiffness matrix of a system. Using screw theory, he defined six principal screw which are the solution to a simple eigenvalue problem. In the 1920s Richard von Mises [2], looked again at the problem. Von Mises was able to describe a system of 15 invariants of the stiffness matrix.

In robotics, Josip Lončarić [3] looked again at these problems in the late 1980s. He described a normal form for the stiffness matrix. That is, almost all spatial stiffness 
matrices can be transformed into a fairly simple shape by applying a suitable rigid transformation.

More recently, Patterson and Lipkin [4, 5] introduced the idea of force-compliant and torque-compliant axes. These were derived from ideas studied by Dimentberg [6] in the 1960s. Further, Patterson and Lipkin, made important contributions to the case where the stiffness matrix determines a stable equilibrium configuration of the rigid body it is supporting.

In some applications designers attempt to synthesize structures with given compliance properties. These desired properties are invariably specified using a stiffness or compliance matrix for the system. A particular example here is the remote-centrecompliance wrist, often used in robotics. Roberts [7] and Huang and Schimmels [8] have studied the problem of synthesizing given stiffness matrices using parallel arrangements of simple springs.

It is also common in robotics to attempt to simulate compliance in software. For example if it is required that the robot's end-effector should follow a path on a surface then some sort of impedance control can be used to manage the contact force between the robot and its environment.

Ideas here are relevant to the study of compliant grasps. In this context Lin, Burdick and Rimon [9] attempted to define a quality measure for a compliant grasp. This was based on the stiffness matrix of the system and some simple geometric properties of the grasped object. Another approach in this area was given by Bruyninckx, Demy and Kumar [10], here the stiffness matrix of the system was combined with an arbitrary inertia matrix to produce invariants. 
All of these applications involve only first order compliance properties again and hence give another argument for understanding the structure of the general compliance matrix.

Helical or coiled springs are basic mechanical components, and hence it is important to understand their compliance properties. Most introductory courses and texts give formulas for the stiffness of these elements. The formulas are used by designers at least for 'rule-of-thumb' computations. The derivation of these formulas is based on Euler-Bernoulli beam theory, which is known to fail for large deflections and exotic material. However, the theory has been verified and used successfully for many applications where small deflections and standard materials dominate.

The textbook examples make further assumptions to simplify the calculations. This is the 'closely coiled' approximation which assumes that the diameter of the helical spring is much greater than its pitch. In this work, this assumption is not made and so springs of arbitrary pitch and diameter can be considered. Standard texts only give the extensional and torsional stiffness of such a spring, but here the full $6 \times 6$ compliance matrix is found. As might be expected the matrix elements depend on the pitch of the spring and hence there are differences between left and right-handed springs. It should be emphasized that the other, standard assumptions, of elementary beam theory still apply. So it may be expected that the results presented here apply to small deflections in standard engineering materials such as steel.

In the research literature the main emphasis seems to be on vibrational properties of helical springs. However, the static displacement properties of these springs is often treated as a preliminary case. In particular Wittrick [11] derives formulas for 
the compliance of helical springs, but only axial torsion and tension are considered. These formula are almost identical to those derived below. In more modern work, for example [12], Wittrick's partial differential equations for bending of helical springs are analyised. These equations are also used in studies of buckling of helical springs, see [13] for example. Modeling the deflection of the spring using partial differential equations could be seen as a local model as opposed to the global (or lumped) model presented here. The geometrical approach used in this work can be extended fairly easily to give a local model of deformation and to include more general constitutive relations between stress and strain, we hope to address this elsewhere.

In essence the method used here is to model the spring as a sequence of infinitesimal straight segments. By knowing the compliance of the infinitesimal elements the compliance for the entire spring can be found by integrating along the helix. Of course in real springs one would have to take account of the geometry of the ends of the spring but here a theoretical result applicable to any length of spring is sought. The method is clearly applicable to more general shapes of beams, in such cases however, the integrals may not be so straightforward.

A key point is that the compliance matrices for the elements must be found in a common coordinate frame. Hence, rigid transformations will be used to transform from frames local to the elements to a global frame. Fortunately, the helix is generated by a one-parameter subgroup of $S E(3)$ which simplifies the computations. This gives a very straightforward derivation for the compliance of the spring, although the matrix manipulations required are rather extensive. The computation is easily accomplished using a symbolic algebra package such as Mathematica or Maple. 
In the final sections of this work the spring is treated as a general spatial compliance element. The principal wrenches and eigen-compliances are studied. Some numerical results are given to show how these vary with the design parameters of the spring.

\subsection{Rigid Body Motions}

The main assumption of Euler-Bernoulli beam theory is that as the beam deforms, the shape of cross sections do not change. Hence, if a coordinate frame is aligned with the cross section and the axis of the beam, the deformation of the beam can be specified by a rigid transformation of the frame. Hence it is important to know how things transform under rigid motions and rigid changes of coordinates.

A twist is a infinitesimal displacement, an element of the Lie algebra of $S E(3)$. In a Cartesian coordinate frame we can partition twists into an angular displacement $\boldsymbol{\omega}$, and a linear displacement $\mathbf{v}$. So the full six-dimensional twist is given by $\mathbf{s}^{T}=\left(\boldsymbol{\omega}^{T}, \mathbf{v}^{T}\right)$. These vectors will also be called screws here. The two 3-vector parts of the screw have different units, in the SI system the angular displacement will be measured in radians and linear displacement will be given in meters. This should not cause any problems as all the operations that will be performed on screws will respect the units.

The displacement of a point $\mathbf{r}$ on the body can be written as $\mathbf{d r}=\mathbf{v}+\mathbf{r} \times \boldsymbol{\omega}$. Alternatively, one could think of a screw as representing the velocity of the rigid body: a combination of the angular velocity and linear velocity of the body.

To each screw one may associate a line in space, the screw axis, and a scalar called the pitch of the screw. A line in space is specified by its direction $\boldsymbol{\omega}$ and its moment $\mathbf{r} \times \boldsymbol{\omega}$, where $\mathbf{r}$ is any point on the line. These are essentially the Plücker coordinates 
of the line. The translational part of the screw $\mathbf{v}$ can be split into a part perpendicular to $\boldsymbol{\omega}$ and a part parallel to $\boldsymbol{\omega}$,

$$
\mathbf{v}=\mathbf{r} \times \boldsymbol{\omega}+p \boldsymbol{\omega}
$$

The line, $\mathbf{l}^{T}=\left(\boldsymbol{\omega}^{T},(\mathbf{r} \times \boldsymbol{\omega})^{T}\right)$ is called the axis of the screw and the scalar $p$ is called the pitch of the screw. The pitch is given by the quantity $p=\boldsymbol{\omega} \cdot \mathbf{v} / \boldsymbol{\omega} \cdot \boldsymbol{\omega}$ so long as $\boldsymbol{\omega} \neq 0$. When $\boldsymbol{\omega}=0$, the screw represents a pure translation and the pitch is said to be infinite. Zero pitch corresponds to pure rotations and positive pitch screws are said to be right handed while negative pitch screws are left handed. The SI units for the pitch are of course, meters.

Now an active transformation will move the screw according to the relation,

$$
\mathbf{s}^{\prime}=H \mathbf{s}=\left(\begin{array}{cc}
R & 0 \\
T R & R
\end{array}\right)\left(\begin{array}{l}
\boldsymbol{\omega} \\
\mathbf{v}
\end{array}\right)
$$

where $R$ is the rotation matrix of the transformation and $T$ is the anti-symmetric matrix representing the translation, $(T \mathbf{x}=\mathbf{t} \times \mathbf{x})$. The transformation matrix $H$ here is an element of the adjoint representation of the group $S E(3)$. To preserve the dimensions of the screws it is clear that the elements of the rotation matrix $R$ must be dimensionless while the elements of the translation matrix $T$ must have dimensions of length, meters in SI units.

Now the corresponding passive transformation, that is a change of coordinates by the same rotation and translation is given by the inverse of the active transformation matrix $H$,

$$
\overline{\mathbf{s}}=H^{-1} \mathbf{s}=\left(\begin{array}{cc}
R^{T} & 0 \\
-R^{T} T & R^{T}
\end{array}\right)\left(\begin{array}{c}
\boldsymbol{\omega} \\
\mathbf{v}
\end{array}\right)
$$

A wrench is also a six-dimensional vector, but this time an element of the dual to 
the Lie algebra. In the same coordinate system, we can partition the wrenches into force and moment vectors, $\mathcal{W}^{T}=\left(\boldsymbol{\tau}^{T}, \mathbf{F}^{T}\right)$. The vector $\mathbf{F}$ is the force and $\boldsymbol{\tau}$ is the moment acting on the rigid body. The units for wrenches are again partitioned, (Nm) for the torque components and $(\mathrm{N})$ for the force components in the SI system.

The generalized momentum of a rigid body, consisting of the linear and angular momentum also form an element of the dual to the Lie algebra. The key difference between Lie algebra elements and elements of the dual space is their transformation properties.

The pairing of a wrench and a screw $\mathcal{W}^{T} \mathbf{s}$, gives a quantity proportional to the energy or power, depending whether the screw represents a displacement or a velocity. Now these quantities are scalars (with SI units J and W respectively), that is they are invariant with respect to rigid transformations. To ensure that the combination is invariant, wrenches must transform under the inverse transpose representation of $H$ for an active transformation and the transpose of $H$ for a coordinate change,

$$
\mathcal{W}^{\prime}=H^{-T} \mathcal{W}=\left(\begin{array}{cc}
R & T R \\
0 & R
\end{array}\right)\left(\begin{array}{l}
\boldsymbol{\tau} \\
\mathbf{F}
\end{array}\right), \overline{\mathcal{W}}=H^{T} \mathcal{W}=\left(\begin{array}{cc}
R^{T} & -R^{T} T \\
0 & R^{T}
\end{array}\right)\left(\begin{array}{l}
\boldsymbol{\tau} \\
\mathbf{F}
\end{array}\right)
$$

The inverse transpose of $H$ is an element of the co-adjoint representation of $S E(3)$.

Wrenches also have axes and pitches, this time the pitch of a wrench $\mathcal{W}^{T}=$ $\left(\boldsymbol{\tau}^{T}, \mathbf{F}^{T}\right)$ is given by, $p=\mathbf{F} \cdot \boldsymbol{\tau} / \mathbf{F} \cdot \mathbf{F}$, and the axis of the wrench is the line with direction, $\mathbf{F}$ and moment $(\boldsymbol{\tau}-p \mathbf{F})$.

More details on the group $S E(3)$ and its applications to robotics and mechanism theory can be found in [14] for example. In the following section we briefly look at stiffness and compliance matrices, this material may be well known but it is the 
transformation properties of these matrices that we want to emphasizes.

\section{Stiffness and Compliance Matrices}

The general setting is as follows. Consider a rigid body subject to a potential field. This potential may have any cause, electrical, magnetic, gravitational and so forth, but here of course, the idea is that it is due to a coiled spring.

\subsection{Stiffness Matrices}

The potential function is a function on the group of rigid body motions $S E(3)$, as the body translates or rotates the potential changes in general. The force and moment can be combined into a wrench as in the previous section. The wrench on the body due to the potential is given by the gradient of the potential as usual,

$$
\mathcal{W}=-d \Phi
$$

This implies that wrenches are cotangent vectors, $d$ is the exterior derivative here. In fact, since the configuration manifold of a rigid body is a Lie group, we can think of wrenches as elements of the dual to the Lie algebra.

At an equilibrium configuration, where $\mathcal{W}=\mathbf{0}$, the Hessian of the potential energy defines a symmetric tensor. This is the stiffness matrix, $K$. The stiffness matrix will be a $6 \times 6$ symmetric matrix. In particular cases where the potential is given explicitly the stiffness matrix can be derived directly from the potential by differentiating, see [15] for example.

The stiffness matrix maps twists to wrenches. Let $\mathbf{s}^{T}=\left(\boldsymbol{\omega}^{T}, \mathbf{v}^{T}\right)$ be a twist or 
screw, that is an element of the Lie algebra of $S E(3)$. To produce a small displacement $\mathbf{s}^{T}=\left(\boldsymbol{\omega}^{T}, \mathbf{v}^{T}\right)$, the wrench that must be applied is given by,

$$
\left(\begin{array}{l}
\boldsymbol{\tau} \\
\mathbf{F}
\end{array}\right)=K\left(\begin{array}{l}
\boldsymbol{\omega} \\
\mathbf{v}
\end{array}\right)
$$

Alternatively, this formula can be interpreted as giving the displacement produced by a specified wrench. This is, of course a general form of Hooke's law.

From the above formula the transformation properties of the stiffness matrix can be inferred. Suppose that the rigid body and the displacement screw undergo a rigid transformation. In these circumstances it should be expected that the wrench produced will be the same as before but transformed by the same group element, that is,

$$
H^{-T} \mathcal{W}=K^{\prime} H \mathbf{s}
$$

From this it is easily seen that $K^{\prime}=H^{-T} K H^{-1}$ for an active transformation. In a similar fashion it can be seen that under a rigid change of coordinates the stiffness matrix will transform according to, $\bar{K}=H^{T} K H$.

The units of the stiffness matrix are partitioned like the screws and wrenches. In the SI system the top left-hand $3 \times 3$ block will have units $\mathrm{Nm}$, whilst the top right and bottom left-hand blocks will have units N. Finally the bottom right-hand $3 \times 3$ block will have units $\mathrm{Nm}^{-1}$. With these choices the units for the screws and wrenches as given by the 'Hooke's law' formula above will be correct.

\subsection{Compliance Matrices}

The compliance matrix of the system is defined as the inverse of the stiffness matrix,

$C=K^{-1}$. Clearly if the stiffness matrix is singular this definition will fail. However, 
in most practical cases $K$ is positive definite and hence non-singular. The displacement of the body and the wrench acting on it are now related by the formula,

$$
C \mathcal{W}=\mathbf{s}
$$

This of course defines the units for the elements of the compliance matrix. As with the stiffness matrix each $3 \times 3$ block will have its own units, $\mathrm{N}^{-1} \mathrm{~m}^{-1}$ for the top left block, $\mathrm{N}^{-1}$ for the top right and bottom left blocks and $\mathrm{N}^{-1} \mathrm{~m}$ for the bottom right block.

For a serial device composed of compliant elements connected in series, such as a robot or a beam, the compliance matrices for the elements add to give the compliance matrix for the whole device. To see why this is so consider a beam or a robot composed of many elements or links connected in series. The $i$-th element will experience a wrench $\mathcal{W}$ due to the action of the $i+1$-th element, the next element along the beam. For equilibrium, this element must also experience a wrench $-\mathcal{W}$ due to the $i-1$-th element. Hence by Newton's third law, the wrench acting on element $i-1$ due to element $i$ will be $\mathcal{W}$. So all the elements along the beam experience the same wrench $\mathcal{W}$ which is the same as the wrench acting on the tip of the beam. Now the displacement of the $i$-th link can be written as $C_{i} \mathcal{W}=\left(\mathbf{s}_{i}-\mathbf{s}_{i-1}\right)$, where $\mathbf{s}_{i}-\mathbf{s}_{i-1}$ gives the relative displacement of the two ends of the element. Supposing the beam is composed of $n$ elements then by adding these relations along the beam, it is easy to see that total relative displacement of the entire beam can be written as,

$$
\left(\sum_{i=1}^{n} C_{i}\right) \mathcal{W}=\left(\mathbf{s}_{n}-\mathbf{s}_{0}\right)
$$

Hence, the compliance matrix for the whole beam is given by the sum,

$$
C=\sum_{i=1}^{n} C_{i}
$$


In beam problems a compliance density $c(\mu)$ can be introduced. Here $\mu$ is the length along the beam and an element of length $\Delta \mu$ will have a compliance matrix, $C_{i}=$ $C(\mu) \Delta \mu$. Proceeding to the limit $\Delta \mu \rightarrow 0$, the compliance matrix for a beam of length $l$ will be given by the integral,

$$
C=\int_{0}^{l} c(\mu) d \mu
$$

It should be noted that both the summation and the integral require that the compliances are given in a common coordinate frame.

Finally here the transformation properties of a compliance matrix are given. This is straightforward due to the relationship with the stiffness matrix. Under an active rigid transformation the compliance matrix becomes, $C^{\prime}=H C H^{T}$. A passive rigid change of coordinates changes the compliance matrix according to, $\bar{C}=H^{-1} \mathrm{CH}^{-T}$.

\subsection{Principal Stiffnesses}

In [1], the idea of principal screws and their eigenstiffnesses was introduced. In essence, the principal screws are the eigenvectors $\mathbf{s}$, of the generalized eigenvalue problem,

$$
K \mathbf{s}=\lambda Q_{0} \mathbf{s}
$$

The characteristic values $\lambda$, are called the eigenstiffnesses of the stiffness matrix $K$. The $6 \times 6$ matrix $Q_{0}$ has the partitioned form, $Q_{0}=\left(\begin{array}{cc}0 & I_{3} \\ I_{3} & 0\end{array}\right)$ where $I_{3}$ is the $3 \times 3$ identity matrix. Note that, since $K \mathbf{s}$ is a wrench, the simple eigenvalue equation, $K \mathbf{s}=\lambda \mathbf{s}$ without the $Q_{0}$ matrix, is not coordinate invariant. The solutions of this problem would be different in different coordinate systems. The matrix $Q_{0}$ converts the screw $\mathbf{s}$ to a wrench, moreover this matrix satisfies $H^{T} Q_{0} H=Q_{0}$ for any proper 
rigid transformation $H$. Hence the eigenstiffnesses defined above will be the same in any coordinate system.

The physical significance of the principal screws is as follows. Suppose the end of the spring is subject to a small displacement determined by a screw $\mathbf{~ s . ~ S u c h ~ a ~ d i s - ~}$ placement will, in general, consist of a small rotation about some axis together with a small translation along the same axis. Now according to Hook's law $\mathcal{W}=K \mathbf{s}$, the small displacement will give rise to a wrench. In general, a wrench consists of a torque about some axis together with a force in the direction of the axis. Now for general displacements the axis of the screw and the axis of the wrench will be different. For principle screws these axes will coincide. Moreover, the pitch of the principle screw $p=\boldsymbol{\omega} \cdot \mathbf{v} / \boldsymbol{\omega} \cdot \boldsymbol{\omega}$ will be the same as the pitch of the wrench it generates $p=\mathbf{F} \cdot \boldsymbol{\tau} / \mathbf{F} \cdot \mathbf{F}$. The corresponding eigenstiffness is a constant of proportionallity. In terms of design the principle screws are perhaps not that useful, general screw motions are rarely required. It was in part for this reason that Patterson and Lipkin introduced the idea of force-compliant and torque-compliant axes in [4]. These are axes where a pure translation produces a pure force in the same direction for example. However, only rather special stiffness matrices have such axes.

It is not difficult to show that the stiffness matrix is completely specified by its set of (in general) six principal screws and their corresponding eigenstiffnesses. The eigenstiffnesses are not all necessarily real, unlike the standards case of eigenvalues of a symmetric matrix. However, if $K$ is positive definite, the eigenstiffnesses are all real and moreover three will be positive and three negative. For more details see [16].

For compliance matrices there is a dual theory, consider the generalized eigenvalue 
equation,

$$
C \mathcal{W}=\lambda Q_{0} \mathcal{W}
$$

The solutions $\mathcal{W}$ will be referred to as principal wrenches of the system and the generalized eigenvalues $\lambda$ will be called eigen-compliances. The matrix $Q_{0}$ has be used to convert a wrench to a screw, perhaps a different notation should have been used for this since although the matrix entries are the same as above, their units will be different, however no confusion should arise. Note that, $H^{-1} Q_{0} H^{-T}=Q_{0}$ for any proper rigid motion $H$ so the eigen-compliances are also invariant with respect to coordinate changes. The physical interpretaion of the principle wrenches is the same as for the principle screws, except that perhaps we would think of the wrench causing the displacement in this case.

Given an invertible stiffness matrix $K$ the principal screws and principal wrenches are simply related. Suppose $\mathbf{s}$ is a principal screw of $K$ with corresponding eigenstiffness $\lambda$, so that,

$$
K \mathbf{s}=\lambda Q_{0} \mathbf{s}
$$

Now the compliance matrix for the system is given by the inverse of $K$ as usual, $C=$ $K^{-1}$, so if the above equation is pre-multiplied by $C$ the result is,

$$
\mathbf{s}=\lambda C Q_{0} \mathbf{s}
$$

Since $Q_{0} Q_{0}=I_{6}$ the $6 \times 6$ identity matrix, this relation can be rewritten as,

$$
C Q_{0} \mathbf{s}=\frac{1}{\lambda} Q_{0} Q_{0} \mathbf{s}
$$

Hence, the eigen-compliance is the reciprocal of the eigenstiffness and the principal wrench corresponding to the principal screw $\mathbf{s}$ is given by $\mathcal{W}=Q_{0} \mathbf{s}$. 
The familiar orthogonality properties of eigenvectors of a symmetric matrix have analogues here. A pair of principal wrenches $\mathcal{W}_{i}$ and $\mathcal{W}_{j}$, with different eigen-compliances will satisfy the relations,

$$
\mathcal{W}_{i}^{T} C \mathcal{W}_{j}=0 \quad \text { and } \quad \mathcal{W}_{i}^{T} Q_{0} \mathcal{W}_{j}=0
$$

Wrenches satisfying the second relation here are said to be reciprocal.

\subsection{Small Elements in Beams}

Consider an arbitrarily shaped slender beam. The beam can be split into short straight elements. In equilibrium, when the wrench at the end of the beam is $\mathcal{W}$ the wrench acting on each element of the beam will also be $\mathcal{W}$, or more precisely a pair of wrenches $\pm \mathcal{W}$

The effect of this stress on the bar is to cause small deflections of the ends of the elements. These small deflections will be measured by screws $\mathbf{s}_{1}$ and $\mathbf{s}_{2}$. The relation between the wrench and the small deformation will be given by Hooke's law,

$$
C \mathcal{W}=\left(\mathbf{s}_{2}-\mathbf{s}_{1}\right)
$$

where $C$ is the compliance matrix of the element.

It must be emphasized here that the coordinate frame used here for simplicity is centred at the middle of the beam element, the $x$-axis is directed along the length of the beam and the $y$ and $z$-axes are perpendicular to length of the beam, see figure 1.It is also convenient to assume that the $y$ and $z$-axes are aligned with the principal bending directions of the beam. 
Von Mises [2] showed that the compliance matrix of a straight beam of length $l$, is given by the diagonal matrix,

$$
C=\left(\begin{array}{cccccc}
\frac{l}{G J} & 0 & 0 & 0 & 0 & 0 \\
0 & \frac{l}{E I_{z}} & 0 & 0 & 0 & 0 \\
0 & 0 & \frac{l}{E I_{y}} & 0 & 0 & 0 \\
0 & 0 & 0 & \frac{l}{E A} & 0 & 0 \\
0 & 0 & 0 & 0 & \frac{l^{3}}{12 E I_{z}} & 0 \\
0 & 0 & 0 & 0 & 0 & \frac{l^{3}}{12 E I_{y}}
\end{array}\right)
$$

The symbols used have their usual meaning $E$ is Young's modulus, $G$ the shear modulus of the material, $A$ is the cross-sectional area of the beam and the $I \mathrm{~s}$ and $J$ are the relevant area integrals. If the length of the beam $l$ is small we can divide by $l$ and then take the limit as $l$ tends to zero to find the compliance density matrix of the beam,

$$
c_{0}=\left(\begin{array}{cccccc}
\frac{1}{G J} & 0 & 0 & 0 & 0 & 0 \\
0 & \frac{1}{E I_{z}} & 0 & 0 & 0 & 0 \\
0 & 0 & \frac{1}{E I_{y}} & 0 & 0 & 0 \\
0 & 0 & 0 & \frac{1}{E A} & 0 & 0 \\
0 & 0 & 0 & 0 & 0 & 0 \\
0 & 0 & 0 & 0 & 0 & 0
\end{array}\right)
$$

This is the compliance density for the element. That is, the compliance matrix for the element is $C=c_{0} \Delta \mu$.

The usual interpretation of the zeros in the 5th and 6th places here is that such a beam is 'unshearable'. Although the beam is infinitesimally unshearable these entries are non-zero for a beam of finite length.

This result may be interpreted as follows, Suppose a force $F_{x}$ is applied along 
the $x$-axis of the beam. If the length of the element is $\Delta \mu$ and the magnitude of the deflection is $\Delta x$, then from elementary mechanics we have,

$$
F_{x} \frac{1}{E A}=\frac{\Delta x}{\Delta \mu}
$$

where $E$ is Young's modulus and $A$ is the cross-sectional area of the beam. The other matrix entries have similar interpretations. For example, consider a simple torsion about the length of the beam. If the torque about the $x$-axis is $\tau_{x}$ and the corresponding angular deflection of the beam is $\Delta \phi_{x}$ then,

$$
\tau_{x} \frac{1}{G J}=\frac{\Delta \phi_{x}}{\Delta \mu}
$$

where $G$ is the shear modulus and $J$ is the polar inertia of the beam.

For pure bending, if a bending moment $\tau_{y}$ is applied about the $y$-axis, the result is an angular deflection $\phi_{y}$,

$$
\tau_{y} \frac{1}{E I_{z}}=\frac{\Delta \phi_{y}}{\Delta \mu}
$$

where $I_{z}$ is the second moment of area for the beam, see figure 1.Clearly a similar result applies to bending about the $z$-axis.

Finally, for forces in the $y$ or $z$ directions, to first order, there will be negligible deflection at the ends of the bar. It must be remembered that these are pure forces, that is, without any bending moment, so the forces must pass through the centre of the bar. This of course is another interpretation of the infinitesimally unshearable assumption. 
Putting all this together we get the equation,

$$
c_{0}\left(\begin{array}{c}
\tau_{x} \\
\tau_{y} \\
\tau_{z} \\
F_{x} \\
F_{y} \\
F_{z}
\end{array}\right)=\frac{1}{\Delta \mu}\left(\begin{array}{c}
\Delta \phi_{x} \\
\Delta \phi_{y} \\
\Delta \phi_{z} \\
\Delta x \\
\Delta y \\
\Delta z
\end{array}\right) .
$$

\section{The Helical Spring}

The helical or coiled spring can be thought of as a simple beam where the unbent shape of the beam is given by a helix. More precisely, the shape of the spring is given by a small element swept along by a simple screw motion. Hence, the compliance matrix for the spring can be found by integrating the compliance densities for the elements along the length of the spring, see figure 2.This implies that there is no super-coiling, that is the cross-section does not turn about its centre as it sweeps along the helix.

\subsection{Small Elements along the Spring}

To build up the spring from small elements we begin with an element crossing the $y$ axis. This is then swept around a helix using the screw motion, a pitch $p$ helix about the $z$-axis.

In terms of an angular parameter $\theta$ the length of the element is given by,

$$
d \mu=\sqrt{r^{2}+p^{2} / 4 \pi^{2}} d \theta
$$

here $r$ is the radius of the helix, that is the distance of the initial element from the origin. 
In the following it will be convenient to abbreviate this, so that $\gamma=\sqrt{r^{2}+p^{2} / 4 \pi^{2}}$ and thus $d \mu=\gamma d \theta$.

Now we must find the compliance matrix of the initial element. In general the compliance matrix, or rather the compliance density, will be given by the coordinate transformation,

$$
c=H^{-1} c_{0} H^{-T}
$$

we have two coordinate systems here, one centred in the middle of the spring, the other centred in the small beam element, see figure 2 .Now $c_{0}$ is the density matrix for the element expressed in the dashed coordinate frame, figure 2again. The form of $c_{0}$ was given in section 2.4 above,(but the coordinates were not dashed there).

The matrix $H$ in the transformation above, is the active transformation which moves the dashed coordinate frame into coincidence with the undashed frame. Rather than find $H$ and then invert, it is simpler to find $H^{-1}$ directly. The transformation $H^{-1}$ is again a rigid transformation, it gives the active transformation from which moves the undashed frame to the dashed one. Since it is a rigid transformation we can partition it into rotation and translation matrices in the usual way,

$$
H^{-1}=\left(\begin{array}{cc}
R & 0 \\
T R & R
\end{array}\right)
$$

where the rotation matrix is about the $y$ axis and the translation is also along the $y$-axis,

$$
R=\left(\begin{array}{ccc}
-r / \gamma & 0 & -p / 2 \pi \gamma \\
0 & 1 & 0 \\
p / 2 \pi \gamma & 0 & -r / \gamma
\end{array}\right) \quad T=\left(\begin{array}{ccc}
0 & 0 & r \\
0 & 0 & 0 \\
-r & 0 & 0
\end{array}\right)
$$

From here is is straightforward but rather tedious to compute the compliance den- 
sity $c=H^{-1} c_{0} H^{-T}$,

$$
c=\left(\begin{array}{cccccc}
x_{11} & 0 & x_{13} & r x_{13} & 0 & -r x_{11} \\
0 & x_{22} & 0 & 0 & 0 & 0 \\
x_{13} & 0 & x_{33} & r x_{33} & 0 & -r x_{13} \\
r x_{13} & 0 & r x_{33} & r x_{33}+4 \pi^{2} r^{2} u & 0 & -r^{2} x_{13}-2 \pi p r u \\
0 & 0 & 0 & 0 & 0 & 0 \\
-r x_{11} & 0 & -r x_{13} & -r^{2} x_{13}-2 \pi p r u & 0 & r^{2} x_{11}+p^{2} u
\end{array}\right)
$$

where,

$$
\begin{aligned}
x_{11} & =\frac{r^{2}}{\gamma^{2} G J}+\frac{p^{2}}{(2 \pi \gamma)^{2} E I_{y}} \\
x_{13} & =\frac{p r}{2 \pi \gamma^{2} E I_{y}}-\frac{p r}{2 \pi \gamma^{2} G J} \\
x_{22} & =\frac{1}{E I_{z}} \\
x_{33} & =\frac{r^{2}}{\gamma^{2} E I_{y}}+\frac{p^{2}}{(2 \pi \gamma)^{2} G J} \\
u & =\frac{1}{(2 \pi \gamma)^{2} E A}
\end{aligned}
$$

The next step is to sweep this around the helix and integrate. The screw motion that generates the helix has the form, $H^{-1}(\theta)=\left(\begin{array}{cc}R & 0 \\ T R & R\end{array}\right)$ with the rotation about the $z$-axis,

$$
R=\left(\begin{array}{ccc}
\cos \theta & -\sin \theta & 0 \\
\sin \theta & \cos \theta & 0 \\
0 & 0 & 1
\end{array}\right) \text {, and } T=\left(\begin{array}{ccc}
0 & -\frac{p \theta}{2 \pi} & 0 \\
\frac{p \theta}{2 \pi} & 0 & 0 \\
0 & 0 & 0
\end{array}\right)
$$

for the translation in the $z$-direction. The computation is straightforward but rather unwieldy. The results are given in the appendix.

In the following few sections some classical results are studied. 


\subsection{Torsional and Extensional Stiffness}

Two classical results for the helical spring are often quoted in text books, the torsional stiffness and the extensional stiffness.

From the above it can be seen that the entry in row 3 column 3 of $c$ is unaffected by the screw motion, hence we can integrate it directly to get the corresponding entry in the compliance matrix for the entire spring. Assuming the limits of integration are $\theta=-\phi / 2$ to $+\phi / 2$ and integrating with respect to $d \mu=\gamma d \theta$ the result

$$
C_{33}=\int_{-\phi / 2}^{\phi / 2} c_{33} \gamma d \theta=\frac{r^{2} \phi}{\gamma E I_{y}}+\frac{p^{2} \phi}{4 \pi^{2} \gamma G J}
$$

is obtained. This corresponds to the torsional compliance of the spring. A torque $\tau$ about the axis of the spring will result in a angular change: $\phi_{z}=C_{33} \tau$. Now in the closely coiled approximation $\gamma \approx r$ and $p / r \approx 0$ so that,

$$
C_{33} \approx \frac{r \phi}{E I_{y}}
$$

Further if the cross section of the spring is assumed to be circular with radius $a$ then $I_{y}=\pi a^{4} / 4$ and

$$
k_{\tau}=1 / C_{33}=\frac{\pi a^{4} E}{4 r \phi}
$$

This agrees with the classical result, see $[17, \S 10-10]$ for example.

The extensional stiffness of the spring gives the extension in the direction of the axis, given a force along the axis. This is simply the reciprocal of the entry in row 6 , column 6 of the compliance matrix. Once again the corresponding entry in the compliance density $c$ found above, doesn't change when swept around the helix, although 
this time it is a little harder to see. The entry is,

$$
c_{66}=\frac{r^{4}}{\gamma^{2} G J}+\frac{r^{2} p^{2}}{4 \pi^{2} \gamma^{2} E I_{y}}+\frac{p^{2}}{4 \pi^{2} \gamma^{2} E A}
$$

So integrating this as above gives,

$$
\int_{-\phi / 2}^{+\phi / 2} c_{66} \gamma d \theta=\frac{r^{4} \phi}{\gamma G J}+\frac{r^{2} p^{2} \phi}{4 \pi^{2} \gamma E I_{y}}+\frac{p^{2} \phi}{4 \pi^{2} \gamma E A}
$$

Using the closely coiled spring approximation as above, the extensional stiffness becomes,

$$
k=\frac{G J}{r^{3} \phi}
$$

For a spring with circular cross section $J=\frac{1}{2} \pi a^{4}$ can be used to get the result,

$$
k=\frac{\pi G a^{4}}{2 r^{3} \phi}
$$

again this agrees with classical texts, see for example [18, p. 27].

In $\S 2$ of [11] Wittrick derives expressions for torsional and extentional compliance of a helical spring. This is done by finding an expression for the potential energy of the spring and then taking derivatives with respect to the axial moment and force. The expressions derived agree almost exactly with the expressions for $C_{33}$ and $C_{66}$ given above. The only difference is the term $p^{2} \phi / 4 \pi^{2} \gamma E A$ which is absent from Wittrick's result because he ignores tension along the wire comprising the spring. Wittrick also gives a result for the 'interaction term' which in our notation would be $C_{36}=C_{63}$, this agrees exactly with the result given in the appendix below. In making these comparisons it should be noted that the 'helix angle' $\alpha$ satisfies $\cos \alpha=r / \gamma$.

The results presented above can be used to investigate the effect of the closely coiled assumption. To do this $\gamma=r \sqrt{1+(p / 2 \pi r)^{2}}$ can be expanded in powers of 
$(p / 2 \pi r)$. Retaining only the first non-closely-coiled term gives,

$$
C_{33}=\frac{r \phi}{E I_{y}}+r \phi\left(\frac{p}{2 \pi r}\right)^{2}\left(\frac{1}{G J}-\frac{1}{2 E I_{y}}\right)+\cdots
$$

and

$$
C_{66}=\frac{r^{3} \phi}{G J}+r \phi\left(\frac{p}{2 \pi r}\right)^{2}\left(\frac{r^{2}}{E I_{y}}-\frac{r^{2}}{2 G J}+\frac{1}{E A}\right)+\cdots
$$

These results show that the difference between the closely coiled results and the results which do not use this simplifying assumption grows approximately quadratically in the small quantity $(p / 2 \pi r)$. Hence, the closely coiled approximations are surprisingly good.

Figure 3 shows a comparison of the closely coiled spring stiffnesses with the formulas given above for $C_{33}$ and $C_{66}$ and quadratic approximations to them. The example used was a steel spring with Young's modulus $0.21 \times 10^{12} \mathrm{~N} \mathrm{~m}^{-2}$ and Poisson ratio 0.3. The spring was assumed to have a radius of $50 \times 10^{-3} \mathrm{~m}$ and a circular cross section of radius $5 \times 10^{-3} \mathrm{~m}$, the length of the spring was taken to be 50 complete turns. It can be seen that the quadratic approximations are extremely good up to large pitches.

\section{The Compliance Matrix for Closely Coiled Springs}

In this section the compliance matrix for the closely coiled spring approximation is studied. Essentially this means that the pitch $p$ is assumed negligible and hence $\gamma \approx r$ the radius of the spring. The results for the elements of the compliance density can be written as,

$$
x_{11} \approx \frac{1}{G J} \quad x_{13} \approx 0 \quad x_{22}=\frac{1}{E I_{z}} \quad x_{33} \approx \frac{1}{E I_{y}} \quad u=\frac{1}{(2 \pi r)^{2} E A}
$$


The compliance matrix is then given by the integral,

$$
C=\int_{-\phi / 2}^{+\phi / 2} H^{-1} c H^{-T} r d \theta
$$

The result obtained is,

$$
C=\left(\begin{array}{cccccc}
X_{1}+X_{2} & 0 & 0 & 0 & 0 & -\frac{2 r^{3}}{G J} \sin \frac{\phi}{2} \\
0 & X_{1}-X_{2} & 0 & 0 & 0 & 0 \\
0 & 0 & \frac{r \phi}{E I_{y}} & \frac{2 r^{3}}{E I_{y}} \sin \frac{\phi}{2} & 0 & 0 \\
0 & 0 & \frac{2 r^{3}}{E I_{y}} \sin \frac{\phi}{2} & X_{3}+X_{4} & 0 & 0 \\
0 & 0 & 0 & 0 & X_{3}-X_{4} & 0 \\
-\frac{2 r^{3}}{G J} \sin \frac{\phi}{2} & 0 & 0 & 0 & 0 & \frac{r^{4} \phi}{G J}
\end{array}\right)
$$

where,

$$
\begin{aligned}
X_{1} & =\frac{r \phi}{2}\left(\frac{r}{G J}+\frac{1}{E I_{z}}\right) \\
X_{2} & =\frac{r}{2}\left(\frac{r}{G J}-\frac{1}{E I_{z}}\right) \sin \phi \\
X_{3} & =\frac{r \phi}{2}\left(\frac{r}{E I_{y}}+\frac{1}{E A}\right) \\
X_{4} & =\frac{r}{2}\left(\frac{r}{E I_{y}}+\frac{1}{E A}\right) \sin \phi
\end{aligned}
$$

It should be noted that if the spring has an integer number of coils, that is if $\phi$ is a multiple of $2 \pi$, then the compliance matrix will be diagonal,

$$
C=\left(\begin{array}{cccccc}
d_{1} & 0 & 0 & 0 & 0 & 0 \\
0 & d_{1} & 0 & 0 & 0 & 0 \\
0 & 0 & d_{2} & 0 & 0 & 0 \\
0 & 0 & 0 & d_{3} & 0 & 0 \\
0 & 0 & 0 & 0 & d_{3} & 0 \\
0 & 0 & 0 & 0 & 0 & d_{4}
\end{array}\right)
$$


where $d_{1}=n r \pi\left(\frac{r}{G J}+\frac{1}{E I_{z}}\right), d_{2}=\frac{2 n r \pi}{E I_{y}}, d_{3}=n r \pi\left(\frac{r}{E I_{y}}+\frac{1}{E A}\right), d_{4}=\frac{2 n r^{4} \pi}{G J}$, and $n$ is the number of coils.

For this diagonal compliance matrix it is very easy to work out the eigen-compliances and principal axes. The principal wrenches of the compliance matrix lie along the coordinate axes and pass through the origin

$$
\begin{aligned}
& \mathcal{W}_{x}^{+}=\left(\begin{array}{c}
\sqrt{d_{3}} \\
0 \\
0 \\
+\sqrt{d_{1}} \\
0 \\
0
\end{array}\right), \mathcal{W}_{y}^{+}=\left(\begin{array}{c}
0 \\
\sqrt{d_{3}} \\
0 \\
0 \\
+\sqrt{d_{1}} \\
0
\end{array}\right), \mathcal{W}_{z}^{+}=\left(\begin{array}{c}
0 \\
0 \\
\sqrt{d_{4}} \\
0 \\
0 \\
+\sqrt{d_{2}}
\end{array}\right) \text {, } \\
& \mathcal{W}_{x}^{-}=\left(\begin{array}{c}
\sqrt{d_{3}} \\
0 \\
0 \\
-\sqrt{d_{1}} \\
0 \\
0
\end{array}\right), \mathcal{W}_{y}^{-}=\left(\begin{array}{c}
0 \\
\sqrt{d_{3}} \\
0 \\
0 \\
-\sqrt{d_{1}} \\
0
\end{array}\right), \mathcal{W}_{z}^{-}=\left(\begin{array}{c}
0 \\
0 \\
\sqrt{d_{4}} \\
0 \\
0 \\
-\sqrt{d_{2}}
\end{array}\right)
\end{aligned}
$$

The pitches of these wrenches are simply,

$$
\pi_{x}^{ \pm}=\pi_{y}^{ \pm}= \pm 2 \sqrt{\frac{d_{3}}{d_{1}}}, \quad \pi_{z}^{ \pm}= \pm 2 \sqrt{\frac{d_{4}}{d_{2}}}
$$

The corresponding eigen-compliances are,

$$
\lambda_{x}^{ \pm}=\lambda_{y}^{ \pm}= \pm \sqrt{d_{1} d_{3}}, \quad \lambda_{z}^{ \pm}= \pm \sqrt{d_{2} d_{4}}
$$

It should be noted that the principal wrenches occur in positive and negative pairs, that is they have the same axes but opposite pitches and eigen-compliances. More- 
over, two of the pairs of principal wrenches have exactly the same pitches and eigencompliances. When the number of coils in the spring is not an integer it may be expected that these symmetries will be broken.

However, even in the case that the number of coils in the spring is not an integer, the compliance matrix has a high degree of symmetry. From the form of the compliance matrix a number of discrete symmetries can be seen. There are two reflection symmetries, one in the $x y$-plane and one in the $y z$-plane, these can be represented by diagonal $6 \times 6$ matrices as follows,

$$
F_{x y}=\left(\begin{array}{cccccc}
1 & 0 & 0 & 0 & 0 & 0 \\
0 & 1 & 0 & 0 & 0 & 0 \\
0 & 0 & -1 & 0 & 0 & 0 \\
0 & 0 & 0 & -1 & 0 & 0 \\
0 & 0 & 0 & 0 & -1 & 0 \\
0 & 0 & 0 & 0 & 0 & 1
\end{array}\right), F_{y z}=\left(\begin{array}{cccccc}
-1 & 0 & 0 & 0 & 0 & 0 \\
0 & 1 & 0 & 0 & 0 & 0 \\
0 & 0 & 1 & 0 & 0 & 0 \\
0 & 0 & 0 & 1 & 0 & 0 \\
0 & 0 & 0 & 0 & -1 & 0 \\
0 & 0 & 0 & 0 & 0 & -1
\end{array}\right)
$$

The action of these symmetries on the compliance matrix is given by,

$$
C=F_{x y}^{T} C F_{x y}, \quad C=F_{y z}^{T} C F_{y z}
$$

where of course $F^{T}=F$. It should be noted that the action of these reflections on the matrix $Q_{0}$ simply reverses its sign, $F^{T} Q_{0} F=-Q_{0}$. The existence of these symmetries implies that the principal wrenches occur in pairs with equal but opposite eigencompliances. Suppose, $\mathcal{W}$ is a principal wrench with eigen-compliance $\lambda$, then $F^{T} \mathcal{W}$ is also a principal wrench but with eigen-compliance $-\lambda$. This may be see from the following,

$$
F^{T} C \mathcal{W}=\lambda F^{T} Q_{0} \mathcal{W}
$$


now since, $F F^{T}=I_{6}$ this can be written as,

$$
F^{T} C F F^{T} \mathcal{W}=\lambda F^{T} Q_{0} F F^{T} \mathcal{W}
$$

and then from the symmetry properties,

$$
C F^{T} \mathcal{W}=-\lambda Q_{0} F^{T} \mathcal{W}
$$

It should be noted that the combination $F_{x y} F_{y z}=F_{y z} F_{x y}$ is a rotation of $\pi$ radians about the $y$-axis.

Again from the form of the compliance matrix, it is clear that one pair of principal wrenches lies along the $y$-axis, their eigen-compliances are $\pm \sqrt{\left(X_{1}-X_{2}\right)\left(X_{3}-X_{4}\right)}$

and their pitches are $\pm 2 \sqrt{\frac{X_{3}-X_{4}}{X_{1}-X_{2}}}$. Clearly when the number of coils is an integer these principal wrenches are simply $\mathcal{W}_{y}^{ \pm}$, as above.

The other principal wrenches will in general be displaced from the positions found for the case where the spring has an integer number of coils. However, from the symmetries it can be seen that the axes of these principal wrenches will all pass through the origin and will lie in the $x z$-plane.

\subsection{Eigen-Compliances for the Coiled Spring}

In the previous section the principal wrenches and eigen-compliances were studied for the closely coiled approximation to the compliance matrix. In this section the compliance matrix for the helical spring are studied without using the closely coiled approximation.

First note that the two reflection symmetries that were found for the closely coiled spring are no longer symmetries of the system. Indeed the two reflections change a 
left-handed helix into a right-handed one and vice versa. Hence, different compliance matrices can be expected for left-handed and right-handed springs. These will be distinguished by the sign of the pitch $p$. However, one symmetry does persist, the rotation of $\pi$ about the $y$-axis. This rotation is represented by a rigid transformation matrix,

$$
H=\left(\begin{array}{cccccc}
-1 & 0 & 0 & 0 & 0 & 0 \\
0 & 1 & 0 & 0 & 0 & 0 \\
0 & 0 & -1 & 0 & 0 & 0 \\
0 & 0 & 0 & -1 & 0 & 0 \\
0 & 0 & 0 & 0 & 1 & 0 \\
0 & 0 & 0 & 0 & 0 & -1
\end{array}\right)
$$

The compliance matrix $C$, will satisfy the relation $C=H C H$ since, $H=H^{-1}=$ $H^{T}$. This imposes some restrictions on the matrix entries of $C$, in fact it implies that some of the entries must vanish. It is straightforward to show that the compliance matrix obeying this rotational symmetry must have the general form,

$$
C=\left(\begin{array}{cccccc}
C_{11} & 0 & C_{13} & C_{14} & 0 & C_{16} \\
0 & C_{22} & 0 & 0 & C_{25} & 0 \\
C_{13} & 0 & C_{33} & C_{34} & 0 & C_{36} \\
C_{14} & 0 & C_{34} & C_{44} & 0 & C_{46} \\
0 & C_{25} & 0 & 0 & C_{55} & 0 \\
C_{16} & 0 & C_{36} & C_{46} & 0 & C_{66}
\end{array}\right)
$$

See the appendix, for formulas for the non-zero elements.

From the above it is possible to say something about the principal wrenches. There will be a pair of principal wrenches aligned with the $y$-axis, their pitches will be equal but opposite, $\pm 2 \sqrt{C_{55} / C_{22}}$ and their corresponding eigen-compliances will be $C_{25} \pm$ 
$\sqrt{C_{22} C_{55}}$. In these coordinates these principal wrenches can be written,

$$
\mathcal{W}_{y}^{+}=\left(\begin{array}{c}
0 \\
\sqrt{C_{55}} \\
0 \\
0 \\
+\sqrt{C_{22}} \\
0
\end{array}\right), \quad \mathcal{W}_{y}^{-}=\left(\begin{array}{c}
0 \\
\sqrt{C_{55}} \\
0 \\
0 \\
-\sqrt{C_{22}} \\
0
\end{array}\right)
$$

The axes for the other principal wrenches will all lie in planes parallel to the $x z$ plane and will meet the $y$-axis. This can be seen by considering the requirement that different principal wrenches must be reciprocal. That is, any other principal wrench $\mathcal{W}$ must satisfy, $\mathcal{W}^{T} Q_{0} \mathcal{W}_{y}^{+}=\mathcal{W}^{T} Q_{0} \mathcal{W}_{y}^{-}=0$.

As a numerical example consider the springs used in figure 3, if the pitch is taken as $0.02 \mathrm{~m}$, then the compliance matrix can be evaluated,

$C=\left(\begin{array}{cccccc}0.18 & 0 & 0 & -3610^{-6} & 0 & 0 \\ 0 & 0.18 & 0 & 0 & -1110^{-3} & 0 \\ 0 & 0 & 0.15 & -9.410^{-6} & 0 & 0.1510^{-3} \\ -3610^{-6} & 0 & -9.210^{-6} & 1810^{-3} & 0 & -3210^{-6} \\ 0 & -0.1110^{-3} & 0 & 0 & 18.10^{-3} & 0 \\ 0 & 0 & 0.1510^{-3} & -3210^{-6} & 0 & 0.510^{-3}\end{array}\right)$

The units here are SI units as given in section 2.2.

The eigen-compliances of this matrix are simple to compute,

$$
\lambda=-0.0570,0.0568,-0.0569,0.0569-0.0086,0.0089,
$$


The corresponding principal wrenches are,

$$
\left(\begin{array}{c}
0.0000 \\
-0.3242 \\
0.0000 \\
0.0000 \\
1.0000 \\
0.0000
\end{array}\right), \quad\left(\begin{array}{l}
0.0000 \\
0.3242 \\
0.0000 \\
0.0000 \\
1.0000 \\
0.0000
\end{array}\right),
$$

corresponding to the first pair of eigen-compliances. These are the expected principal wrenches along the $y$-axis. The units are as given in section 1.1.

Corresponding to the second pair of eigen-compliances are the two principal wrenches,

$$
\left.\left(\begin{array}{r}
-0.3242 \\
0.0000 \\
0.0006 \\
1.0000 \\
0.0000 \\
-0.0013
\end{array}\right), \quad \begin{array}{r}
0.3242 \\
0.0000 \\
-0.0006 \\
1.0000 \\
0.0000 \\
-0.0013
\end{array}\right) .
$$

The axes of both these principal wrenches are almost aligned with the $x$-axis. As predicted they lie in planes parallel to the $x y$-plane, in fact the axes are very close to passing through the origin. The last pair of principal wrenches are,

$$
\left(\begin{array}{r}
-0.0001 \\
0.0000 \\
-0.0569 \\
0.0017 \\
0.0000 \\
1.0000
\end{array}\right), \quad\left(\begin{array}{l}
0.0001 \\
0.0000 \\
0.0569 \\
0.0018 \\
0.0000 \\
1.0000
\end{array}\right) .
$$


This time the axes are very close to the $z$-axis. As expected there are three positive and three negative eigen-compliances. Also the pitch of each principal wrenches has the same sign as its corresponding eigen-compliance.

It is possible to plot graphs of the eigen-compliances against the pitch of the spring or against the total length of the spring. In figure 4 the eigen-compliances are plotted against the length of the spring. The length of the spring is expressed as an angle giving the number of turn in the helix. Figure 5 shows the variation in the eigen-compliances against the pitch of the spring. Each graph shows a pair of eigen-compliances, each pair is almost symmetrical about the horizontal axis. In both figures the values for the eigencompliances close to the $x$-axis and those close to the $y$-axis are similar in magnitude but those near the $z$-axis are an order of magnitude smaller. As one might expect the compliance of the spring increases with increasing length and with increasing pitch of the spring.

\section{Conclusions}

In this work the compliance matrix for a helical spring has been extensively studied. The only assumptions made were the usual assumptions of standard Euler-Bernoulli beam theory. Using some group theory it was possible to compute the compliance matrix reasonably simply; certainly more simply than in standard texts. It was possible to complete the computations without having to make the usual closely coiled spring approximation. Nevertheless, it has been shown how the results found can be reduced to the standard ones by reintroducing the closely coiled approximation. 
Euler-Bernoulli beam theory was introduced something like two centuries ago and since this time it was been widely used. The closely coiled assumption for helical springs is introduced in standard texts to make computations easier. Hence, it should be expected that the work presented here has the same range of validity as standard Euler-Bernoulli theory, that is to small deflections in beams made of standard materials such as steel. However, better agreement with experiment than the closely coiled results should be expected.

In section 3.2 explicit comparisons were made between the results derived here and those given in standard texts which include the closely coiled assumption. The comparisons were between certain matrix elements of the compliance matrix. A better form of comparison might be between eigen-compliances in either case. This is because the eigen-compliances are invariants of the compliance matrix and hence should be easier to measure in practice. Implicit in most standard texts is the idea that a force (or torque) along the axis of the spring produces only a translation (respectively a twist) about that axis. Even with the closely coiled assumption this is not true.

The methods used in this work could be extended to other beams with different unbent shapes and even with cross sections that change along the length of the beam. The helical spring is an important theoretical example, for more prosaic example one would probably want more than the small deflection properties. To cope with larger deflections Euler-Bernoulli theory must be extended. This was achieved around the turn of the 20th century and is usually known as Cosserat theory, see [19]. The screw description of Euler-Bernoulli theory given here can also be extended to describe the Cosserat theory. The main assumption here is that the state of the beam can be described by a 
sequence of rigid transformations. In other words that the shape of the cross section doesn't alter. For even larger deflections or more exotic materials one must resort to the full theory of elasticity. However even this, much used theory, might benefit from a group theory description. The relevant group is no longer the group of rigid motions but probably the affine group.

\section{References}

[1] R.S. Ball. The Theory of Screws. Cambridge University Press, Cambridge, 1900.

[2] R. von Mises. Motorrechnung, ein neues hilfsmittel in der mechanik. Zeitschrift für Angewandte Mathematik und Mechanik, 1924;4(2):155-181. English Translation by E.J. Baker and K. Wohlhart. Motor Calculus, a New Theoretical Device for Mechanics. Institute for Mechanics, University of Technology Graz, Austria, 1996.

[3] J. Lončarić. Normal forms of stiffness and compliance matrices. IEEE J. of Robotics and Automation, 1987;RA-3(6):567-572.

[4] T. Patterson and H. Lipkin. Structure of robot compliance. J. Mechanical Design, 1993;115:576-580.

[5] T. Patterson and H. Lipkin. A classification of robot compliance. J. Mechanical Design, 1993;115:581-584. 
[6] F.M. Dimentberg. Screw Calculus and its Applications in Mechanics. Izd. Nauka, Moscow, 1965. English translation, Foreign Technology Division, WrightPatterson Air Force Base, Ohio, Document No. FTD-HT-23-1632-67, 1968.

[7] R.G. Roberts. Minimal realization of spatial stiffness matrix with simple springs connected in parallel. IEEE Trans. on Robotics and Automation, 1999;15(5):953958.

[8] S. Huang and J.M. Schimmels. The bounds and realization of spatial stiffnesses achieved with simple springs connected in parallel. IEEE Trans. on Robotics and Automation, 1998;14(3):466-475.

[9] J.W. Burdick Q. Lin and E. Rimon. A quality measure for compliant grasps and fixtures using stiffness characterization. IEEE Trans. on Robotics and Automation, 2000;16(6):675-688.

[10] S. Demy H. Bruyninckx and V. Kumar. Generalized stability of compliant grasps. In IEEE Conference on Robotics and Automation, Leuven, Belgium, 1998. pp. $2396-2402$.

[11] W.H. Wittrick. On elastic wave propogation in helical springs. Int. J. Mech. Sci. $1966 ; 8: 25-47$.

[12] J.E. Mottershead. Finite elements for dynamical analysis of helical rods. Int. J. Mech. Sci. 1980;22:267-283.

[13] L.E. Becker and W.L. Cleghorn. On the buckling of helical compression springs. Int. J. Mech. Sci. 1992;34(4):275-282. 
[14] J.M. Selig. Geometrical Methods in Robotics. Springer Verlag, New York, 1996.

[15] J.M. Selig. The Spatial Stiffness Matrix from Simple Stretched Springs. IEEE Conference on Robotics and Automation, San Francisco, 2000. pp.3314-3319.

[16] J.M. Selig and X. Ding. Structure of the Spatial Stiffness Matrix. IJ Robotics and Automation, vol. 17 No. 1 pp. 1-16 2002.

[17] J.E. Shigley and L.D. Mitchell. Mechanical Engineering Design, 4th ed. McGraw-Hill, New York, 1983.

[18] J.P. Den Hartog. Strength of Materials Dover Publications, New York, 1977.

[19] S. Antman. Nonlinear Problems of Elasticity Springer Verlag, Berlin Applied Mathematical Sciences 107, 1995.

\section{Appendix-Compliance Matrix Elements}

In this appendix the non-zero elements of the compliance matrix are recorded. Here $C$ is the compliance matrix for a helical spring of pitch $p$. The quantities $\gamma, x_{i j}$ and $u$ are as defined in section 3.1. The angle $\phi$ effectively specifies the length of the spring, that is it gives the number of turns in the spring.

$$
\begin{aligned}
C_{11} & =\frac{\gamma \phi}{2}\left(x_{11}+x_{22}\right)+\frac{\gamma}{2} \sin \phi\left(x_{11}-x_{22}\right) \\
C_{13} & =2 \gamma x_{13} \sin \frac{\phi}{2} \\
C_{22} & =\frac{\gamma \phi}{2}\left(x_{11}+x_{22}\right)-\frac{\gamma}{2} \sin \phi\left(x_{11}-x_{22}\right)
\end{aligned}
$$




$$
\begin{aligned}
& C_{33}=\gamma \phi x_{33} \\
& C_{14}=\frac{\gamma r}{2}(\phi+\sin \phi) x_{13}-\frac{p \gamma}{8 \pi}(\sin \phi-\phi \cos \phi)\left(x_{11}-x_{22}\right) \\
& C_{16}=-2 \gamma r x_{11} \sin \frac{\phi}{2} \\
& C_{25}= \frac{\gamma r}{2}(\phi-\sin \phi) x_{13}+\frac{p \gamma}{8 \pi}(\sin \phi-\phi \cos \phi)\left(x_{11}-x_{22}\right) \\
& C_{34}= 2 r \gamma x_{33} \sin \frac{\phi}{2}-\frac{p \gamma}{2 \pi} x_{13}\left(2 \sin \frac{\phi}{2}-\phi \cos \frac{\phi}{2}\right) \\
& C_{36}=-r \gamma \phi x_{13} \\
& C_{44}= \frac{\gamma}{4}\left(r x_{33}+4 \pi^{2} r^{2} u\right)(2 \phi+\sin \phi)-\frac{\gamma p r}{\pi}(\sin \phi-\phi \cos \phi)+\frac{\gamma p^{2}}{96 \pi} \phi^{3}\left(x_{11}+x_{22}\right)- \\
& \quad \quad \frac{\gamma p^{2}}{16 \pi}\left(\frac{\phi-1}{2} \sin \phi+\phi \cos \phi\right)\left(x_{11}-x_{22}\right) \\
& C_{46}=\frac{\gamma p r}{\pi}\left(\sin \frac{\phi}{2}-\frac{\phi}{2} \cos \frac{\phi}{2}\right) x_{11}-2 \gamma \sin \frac{\phi}{2}\left(r^{2} x_{13}+2 \pi p r u\right) \\
& C_{55}=\frac{\gamma}{2}(\phi-\sin \phi)\left(r x_{33}+4 \pi^{2} r^{2} u\right)+\frac{\gamma p r}{\pi}(\sin \phi-\phi \cos \phi) x_{13}+\frac{\gamma p^{2}}{96 \pi} \phi^{3}\left(x_{11}+x_{22}\right)+ \\
& \quad \frac{\gamma p^{2}}{16 \pi}\left(\frac{\phi-1}{2} \sin \phi+\phi \cos \phi\right)\left(x_{11}-x_{22}\right) \\
& C_{66}=\gamma \phi\left(r^{2} x_{11}+p^{2} u\right)
\end{aligned}
$$




\section{Vitae}

Xilun Ding received his B. Eng. degree in mechanical engineering from Zhengzhou Institute of Technology, China in 1991. He then went to Harbin Institute of Technology where he gained an M. Eng. in mechanical engineering in 1994, and a Ph. D. in mechatronics and automation in 1997. He worked at the Robotics Research Institute of Beijing University of Aeronautics and Astronautics as a postdoctoral research fellow from 1997 to 1998. In 1998 Dr. Ding became an Associate Professor at the Beijing University of Aero. \& Astro. He was a senior visiting scholar at the Academy of Mathematics and Systems Sciences, Chinese Academy of Sciences from September 1999 to January 2000. During 2001, he was a postdoctoral research fellow at the School of Computing, Information Systems and Mathematics, South Bank University, London, UK. His research interests include the dynamics of compliant robots, nonholonomic control of space robots and cooperative control of dual redundant manipulators with multi-fingered hand systems.

Jon Selig graduated from the University of York, with a B.Sc. in Physics in 1980. He went to study in the Department of Applied Maths. and Theoretical Physics at the University of Liverpool and was awarded a Ph. D. in 1984. From 1984 to 1987 he was a postdoctoral research fellow in the design discipline of the Open University, studying robot gripping. He joined the Department of Electrical and Electronic Engineering at South Bank Polytechnic in 1987. In 1992 the polytechnic became a university and in 1999 Dr. Selig transferred to his current post in the School of Computing, Information Systems and Mathematics. His research interests can be summarized as the 
applications of modern geometry to problems in robotics. 


\title{
Figure Captions
}

\author{
1. A Small Element in the Beam
}

2. The Helical Spring

3. Graphs of stiffness against the pitch of the spring. In both cases the horizontal line gives the classical result, the lower curve is the result predicted by the theory and the upper curve is the quadratic approximation as given in the text. The material properties are those of steel as given in the text.

4. Graphs of eigen-compliances against the length of the spring (expressed as an angle). The material properties are those of steel as given in the text, with pitch $0.02 \mathrm{~m}$.

5. Graphs of eigen-compliances against pitch. Again the material properties are those of steel as given in the text but with a spring of length 50 turns. 


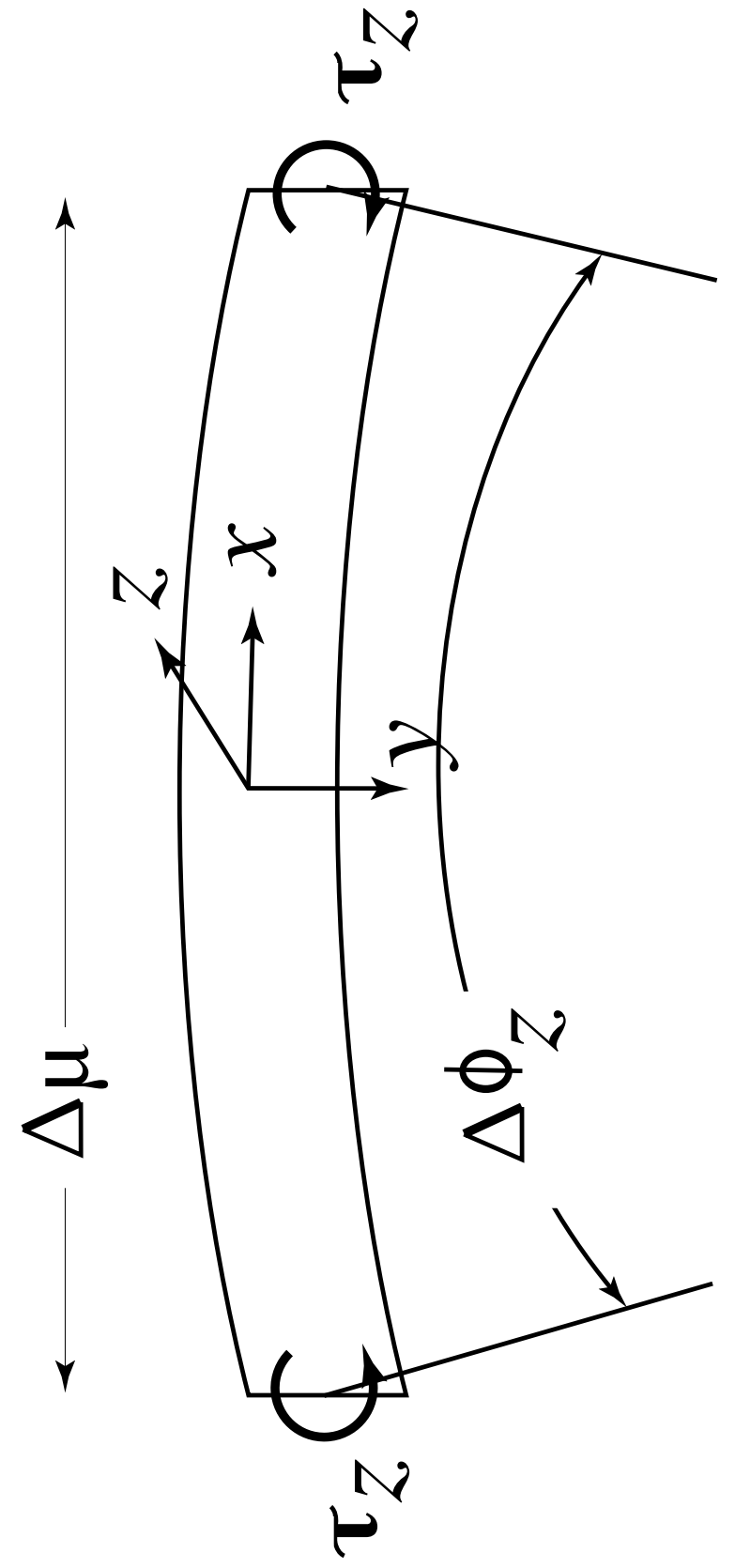




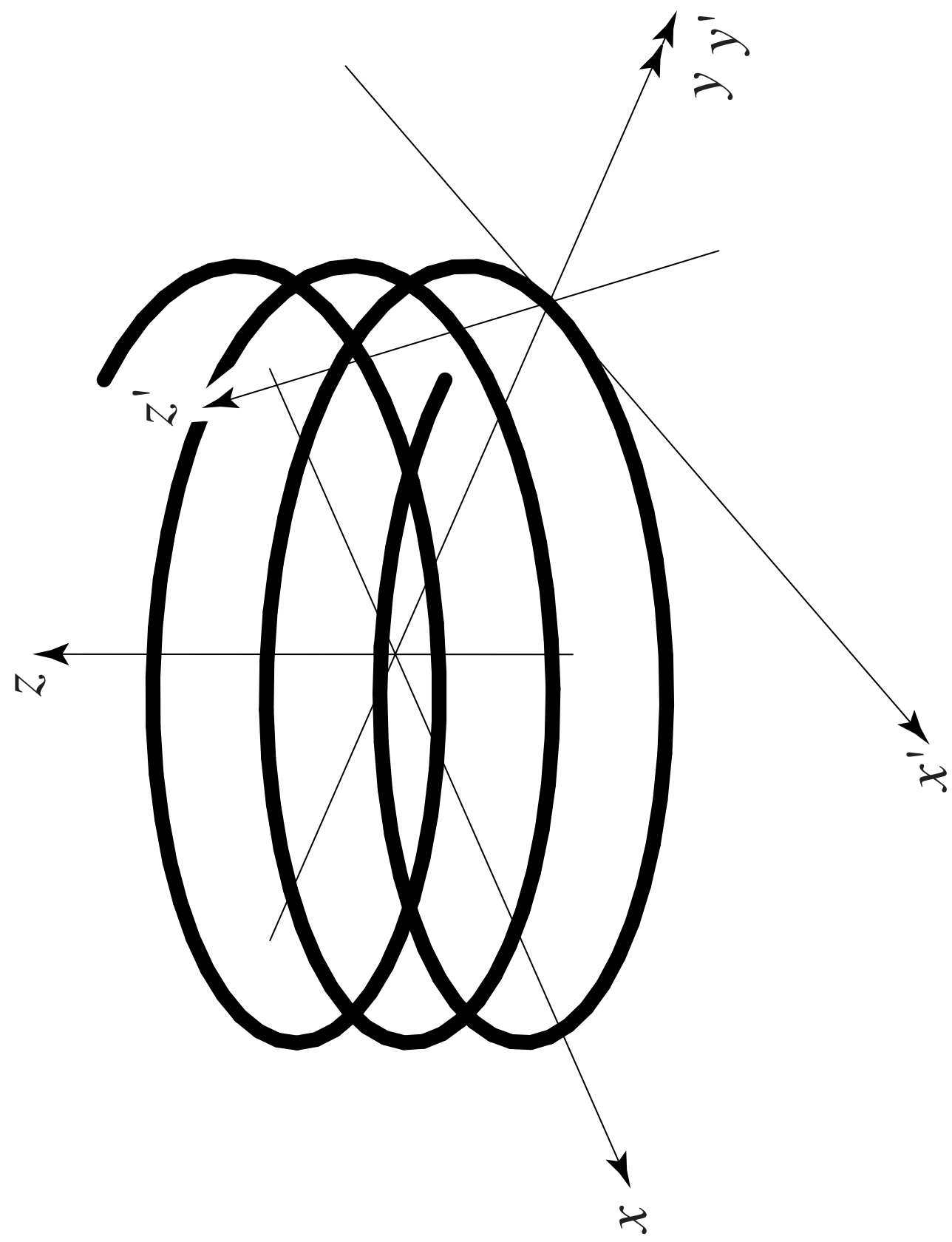



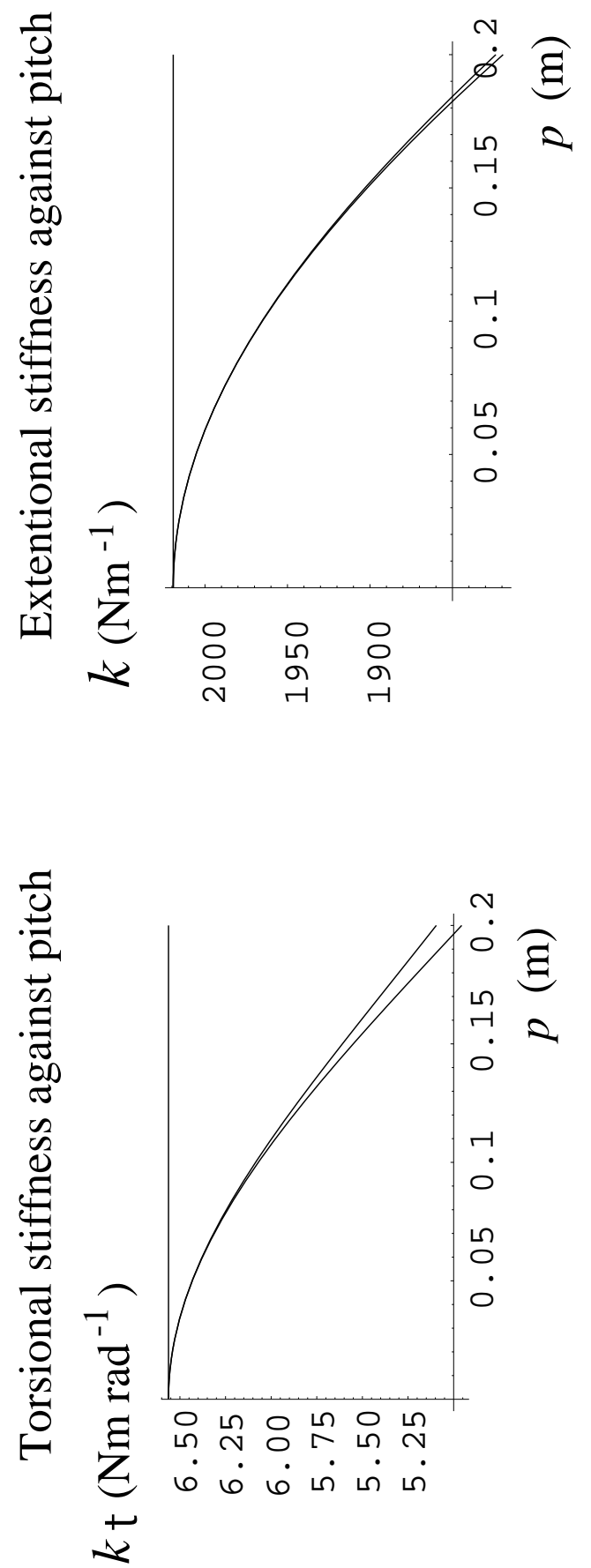

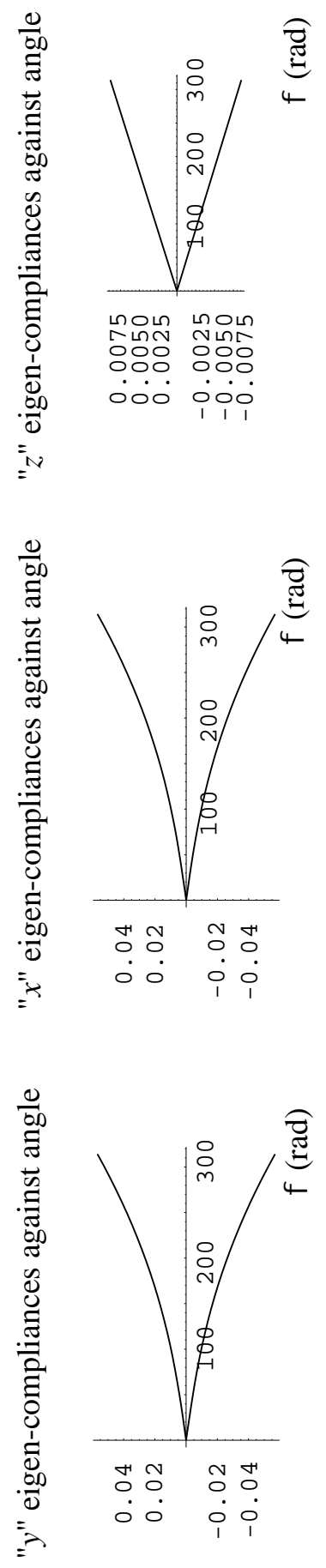

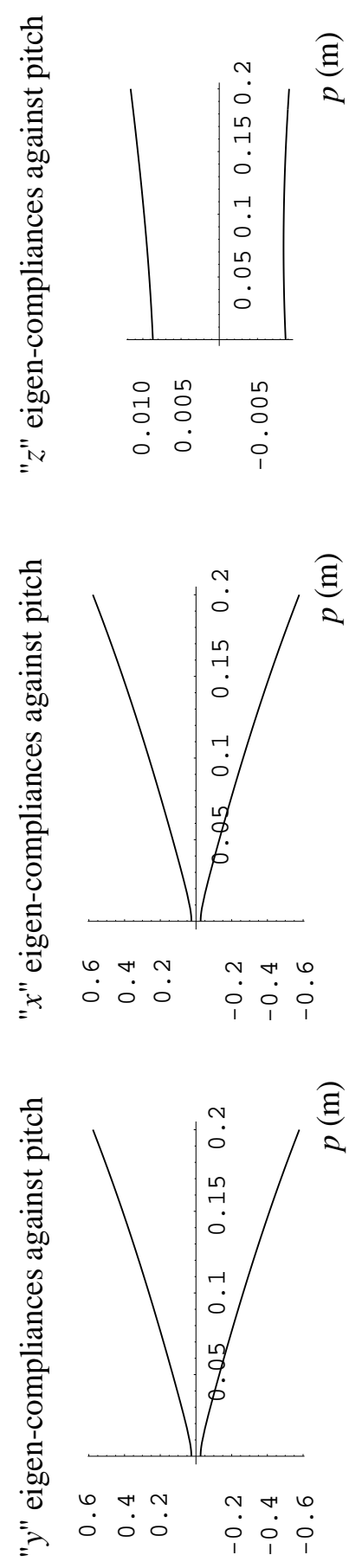


\section{Symbols}

$S E(3)$ The group of proper Euclidean motions in 3 dimensions, that is rigid body motions.

$I_{3}$ The $3 \times 3$ identity matrix.

$R$ A $3 \times 3$ rotation matrix.

$T$ A $3 \times 3$ anti-symmetric matrix representing a translation, so that $T \mathbf{x}=\mathbf{t} \times \mathbf{x}$ for any vector $\mathbf{x}$.

$H$ A $6 \times 6$ matrix representing a rigid transformation, an element of the adjoint representation of $S E(3)$.

s A twist or screw, an element of the Lie algebra se(3).

$\mathcal{W} 6 \times 1$ vector representing a wrench, that is an element of the vector space dual to the Lie algebra $s e(3)$.

$K 6 \times 6$ stiffness matrix.

C $6 \times 6$ compliance matrix.

$E$ Young's modulus.

$G$ The shear modulus.

$A$ The cross sectional area of a beam.

$J$ The polar moment of area.

$I_{x}$ The second moment of area about the $x$-axis. 